\title{
Soil bacterial communities respond differently to graphene oxide and reduced graphene oxide after 90 days of exposure
}

\author{
Junjie $\mathrm{Du}^{1,3}$, Qixing Zhou ${ }^{2, *}$, Jianhu $\mathrm{Wu}^{1}$, Guifeng $\mathrm{Li}^{1}$, Guoqin $\mathrm{Li}^{1}$, Yongning $\mathrm{Wu}^{3}$ \\ 1 College of Food Science, Shanxi Normal University, Linfen 041004, China \\ 2 Key Laboratory of Pollution Processes and Environmental Criteria (Ministry of Education), Tianjin Key Laboratory of Environmental \\ Remediation and Pollution Control, College of Environmental Science and Engineering, Nankai University, Tianjin 300071, China \\ 3 NHC Key Laboratory of Food Safety Risk Assessment, China National Center for Food Safety Risk Assessment, Beijing 100022, China
}

\section{ARTICLE INFO}

\section{Article history:}

Received March 10, 2020

Revised April 18, 2020

Accepted May 20, 2020

\section{Keywords:}

Bacterial community

Biogeochemical cycle

Nanomaterials

Soil functions

\begin{abstract}
A B S T R A C T
Graphene-based nanomaterials (GBNs) are likely to be entering the soil environment in increasing amounts via consumer products. However, the disturbance of bacterial communities and their associated ecological functions by GBNs remains elusive. We performed a soil incubation experiment with the addition of graphene oxide $(\mathrm{GO})$ and reduced graphene oxide (RGO). The Illumina sequencing technique was used to investigate changes in bacterial communities, and the functional groups of the communities were analyzed using the functional annotation of prokaryotic taxa database. After 90 days of exposure, RGO induced a lower bacterial richness than GO. However, GO induced larger changes in community composition and functions than RGO. After exposure to GBNs, some of the functional groups associated with organic matter degradation and biogeochemical cycling of nitrogen and sulfur decreased. However, the functional group associated with aromatic compound degradation increased, possibly because GBNs contain rich aromatic hydrocarbon structures, which are tolerated by this functional group.
\end{abstract}

() Higher Education Press 2020

\section{Introduction}

Soil ecosystems are the final destination of various nanomaterials. The rapidly increasing production and agricultural application of graphene-based nanomaterials (GBNs) will likely lead to their accumulation in the soil environment (Wang et al., 2014; Pulizzi, 2019), and the concentrations of GBNs in agricultural applications have already reached the level of parts per million (ppm) (Yin et al., 2018; Kah et al., 2019). The

\footnotetext{
* Corresponding author

E-mail address: zhouqx@nankai.edu.cn (Q. Zhou)
}

emergence of GBNs poses a threat of disturbing microbial communities, whose metabolism powers biogeochemical cycling in the Earth's ecosystems (Louca et al., 2016). Previous studies have primarily focused on the effects of GBNs on microbial biomass, diversity, and populations, but the results remain inconclusive (Ren et al., 2018). Minor differences in experimental conditions likely shaped some of the observed taxonomic variations. However, the functional groups of microbial communities are more stable than their phylogeny (Gibbons, 2017). This study aimed to analyze the effects of two of the most important GBNs, reduced graphene oxide (RGO) and graphene oxide (GO), on the diversity and functions of soil bacterial communities. 


\section{Materials and methods}

\subsection{Microcosm experiment}

Graphene oxide (XFNANO Materials, Nanjing, China; Diameter $0.5 \mu \mathrm{m}-5 \mu \mathrm{m}$, purity $>99 \mathrm{wt} \%$, carbon $71.23 \%$, oxygen $28.77 \%$, synthesized by Hummers) and RGO (XFNANO Materials, Nanjing, China; Diameter $0.5 \mu \mathrm{m}-5 \mu \mathrm{m}$, purity $>99$ wt $\%$, carbon $95.97 \%$, oxygen $4.03 \%$, synthesized through the complete reduction of $\mathrm{GO}$ ) were applied to soil in suspension at a target concentration of $50 \mathrm{mg} \mathrm{kg}^{-1} \mathrm{dry}$ soil (Rodrigues et al., 2013). The characterizations of these GBNs are presented in the Supporting Information (SI, Figs. S1 and S2). Homogeneous $\mathrm{GO}$ and RGO suspensions of $1.0 \mathrm{~g} \mathrm{~L}^{-1}$ in de-ionized (DI) water was prepared using ultrasonication.

Clean topsoil, which was categorized as cinnamon soil according to Chinese soil taxonomy, was collected from farmland in Linfen of Shanxi, China, with a $\mathrm{pH}$ of 8.14 and containing $20.6 \mathrm{~g} \mathrm{~kg}^{-1}$ organic matter, $1.54 \%$ nitrogen, $1.11 \%$ phosphorus and $6.21 \%$ potassium. The collected soil was airdried below $33^{\circ} \mathrm{C}$ and sieved using a $2 \mathrm{~mm}$ mesh to remove stones and undecomposed debris. Samples of $1.5 \mathrm{~kg}$ dry soils were placed in nine plastic pots for 30 days of preculture at 25 $\pm 1^{\circ} \mathrm{C}$ and with $50 \%$ water holding capacity to reactivate soil microorganisms. Then, the previously prepared GO and RGO suspensions were separately added to different pots of soils and fully mixed with the soil. Equal volumes of DI water were added to soils in the control pots. Although the specific concentrations of GBNs in the soil environment are unknown, the exposure dose chosen in this study was based on previous publications and the development of growing industries (Yin et al., 2018). The culture experiments were performed in a greenhouse at $25 \pm 1{ }^{\circ} \mathrm{C}$ for 90 days, with a $50 \%$ water holding capacity. All treatments were conducted in triplicate.

\subsection{Soil bacterial community analysis}

The illumina sequencing technique was used to investigate changes in the soil bacterial community. Bacterial DNA was extracted from $0.5 \mathrm{~g}$ of the triplicate soil samples using FastDNA $^{\mathrm{TM}}$ Spin extraction kits. The $\mathrm{V} 3$ and $\mathrm{V} 4$ regions of $16 \mathrm{~S}$ rRNA were PCR-amplified using primers of $338 \mathrm{~F}$ and $806 \mathrm{R}$.

The PCR amplicons were purified using Agencourt AMPure Beads and quantified using a PicoGreen dsDNA assay kit. After the individual quantification step, amplicons were pooled in equal amounts, and pair-end $2 \times 300$ bp sequencing was performed using the Illlumina MiSeq platform with MiSeq Reagent Kit v3 from Shanghai Personal Biotechnology Co., Ltd. The data of raw reads were submitted to the National Center for Biotechnology Information database (PRJNA552951)

The QIIME (version 1.8.0) pipeline was employed to process the sequencing data. Then, the low-quality sequences were filtered out, and we clustered sequences into operational taxonomic units (OTUs) with a $97 \%$ similarity using UCLUST. An OTU taxonomic classification was conducted using the Basic Local Alignment Search Tool and Greengenes database. Chao1, ACE, and Shannon indices were calculated using the OTU table in QIIME. The structural variation of bacterial communities across samples was analyzed using UniFrac distance metrics and visualized using principal coordinate analysis (PCoA). The functional groups in the bacterial community were predicted using the FAPROTAX database, which describes the 90 functional groups involved in the main biogeochemical cycling of carbon, nitrogen, and sulfur (Louca et al., 2016). The FAPROTAX database is designed to map prokaryotic taxa to established metabolic or other ecologically relevant functions based on the functional descriptions of cultured strains reported in the peer-reviewed literature (Louca et al., 2016). The detailed procedures of sequencing and analysis were performed at Personal Biotechnology Co., Ltd. and are presented in the SI.

\subsection{Statistical analysis}

All treatments were performed in triplicate. The error bars in the results indicate the standard deviation for three separate experiments (mean $\pm S D$ ). Differences were identified to be statistically significant at $p<0.05$. IBM Statistics SPSS (19.0) was used to analyze the data with a one-way analysis of variance followed by a least significant difference test for comparison.

\section{Results and discussion}

\subsection{Changes in alpha diversity of the bacterial community}

A total of 391821 high-quality sequences were obtained from the nine samples from the three groups. The average number of sequences were 44421,47867 , and 38318 for the "control," "GO," and "RGO" groups, respectively. There were no significant differences $(p>0.05)$ in species diversity, indicated by the Shannon index, between the "control," "GO," and "RGO" groups (Table 1). However, species richness, which was indicated by the ACE and Chao1 indices was significantly $(p<0.05)$ lower in the "RGO" treatment than the "control" and "GO" treatments, demonstrating that RGO exposure induced a lower species richness than GO exposure (Table 1).

Table 1 Richness and diversity of the soil bacterial communities.

\begin{tabular}{llllr}
\hline Treatment Sequence & \multicolumn{3}{c}{$3 \%$ distance } \\
\cline { 2 - 5 } & & ACE & Chao1 & \multicolumn{1}{c}{ Shannon } \\
\hline Control & $44421 \pm 1542 a b$ & $3803 \pm 301 \mathrm{a}$ & $3461 \pm 283 \mathrm{a}$ & $10.36 \pm 0.46 \mathrm{a}$ \\
GO & $47867 \pm 7348 \mathrm{a}$ & $3777 \pm 709 \mathrm{a}$ & $3597 \pm 594 \mathrm{a}$ & $9.58 \pm 1.46 \mathrm{a}$ \\
RGO & $38318 \pm 1036 \mathrm{~b}$ & $2349 \pm 107 \mathrm{~b}$ & $2318 \pm 53 \mathrm{~b}$ & $10.32 \pm 0.37 \mathrm{a}$ \\
\hline
\end{tabular}

Mean values \pm standard error $(n=3)$ followed by different lowercase letters within the same column indicating significant differences at $p<0.05$ using the least significant difference test. 


\subsection{Changes in soil bacterial community composition}

The soil bacterial communities were dominated by Proteobacteria, Actinobacteria, Chloroflexi, Acidobacteria, Gemmatimonadetes, Cyanobacteria, Firmicutes, Nitrospirae, Tectomicrobia, Bacteroidetes, Planctomycetes, and Parcubacteria (Fig. S3). There were no changes in the dominant phyla due to exposure to GO or RGO.

The individual points on the PCoA biplot fell into three distinct clusters, suggesting that the changes in bacterial community structure were evident after exposure to GBNs (PERMANOVA, $p<0.05$; Fig. 1). The "GO" and "control" groups were separated by the PCoA 1 axis (30.23\%), while the "RGO" and "control" groups were separated by the PCoA 2 axis $(18.56 \%)$. These results indicate that RGO exposure induced smaller changes in bacterial community composition than GO.

\subsection{Changes in functional groups of the bacterial community}

In total, 74 functional groups were identified in at least one of the samples (Table S1). Of these, the relative abundances of 11 functional groups were significantly decreased $(p<0.05)$ due to $\mathrm{GO}$ exposure (Fig. 2B-I). These functional groups are associated with the processes of the nitrogen cycle (e.g., ureolysis, nitrogen fixation, nitrate respiration and reduction, and nitrogen respiration), sulfur cycle (e.g., the respiration of sulfur, sulfate, and sulfur compounds), and organic matter degradation (e.g., chemoheterotrophy and fermentation). However, there were only four functional groups that decreased significantly $(p<0.05)$ due to RGO exposure (Fig. $2 \mathrm{~F}, \mathrm{H}, \mathrm{I}$ and $\mathrm{K}$ ): the functional groups involved in nitrate respiration, the respiration of sulfate and sulfur compounds,

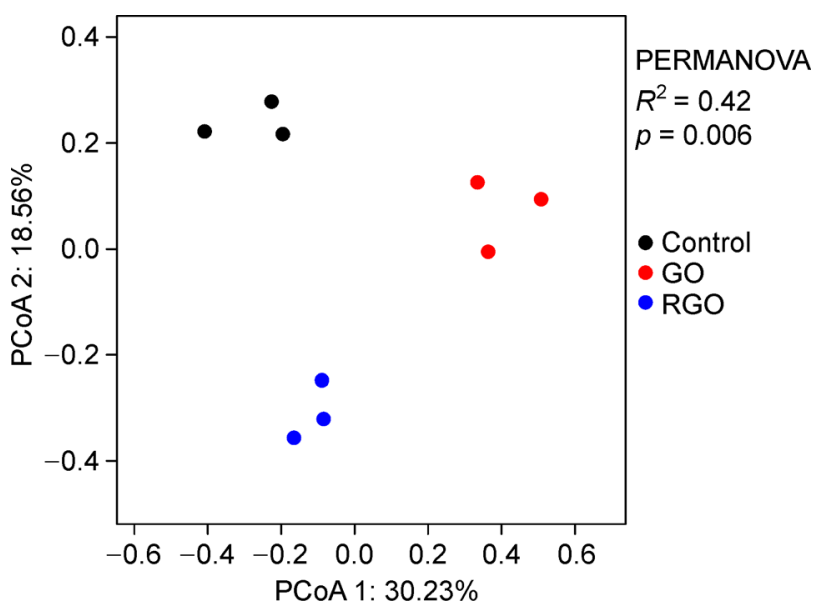

Fig. 1 Principal coordinate analysis plot showing differences in bacterial community structure. The significance of differentiation $(p<0.05)$ of the bacterial community among groups was assessed using permutational multivariate analysis of variance (PERMANOVA, 999 permutations).

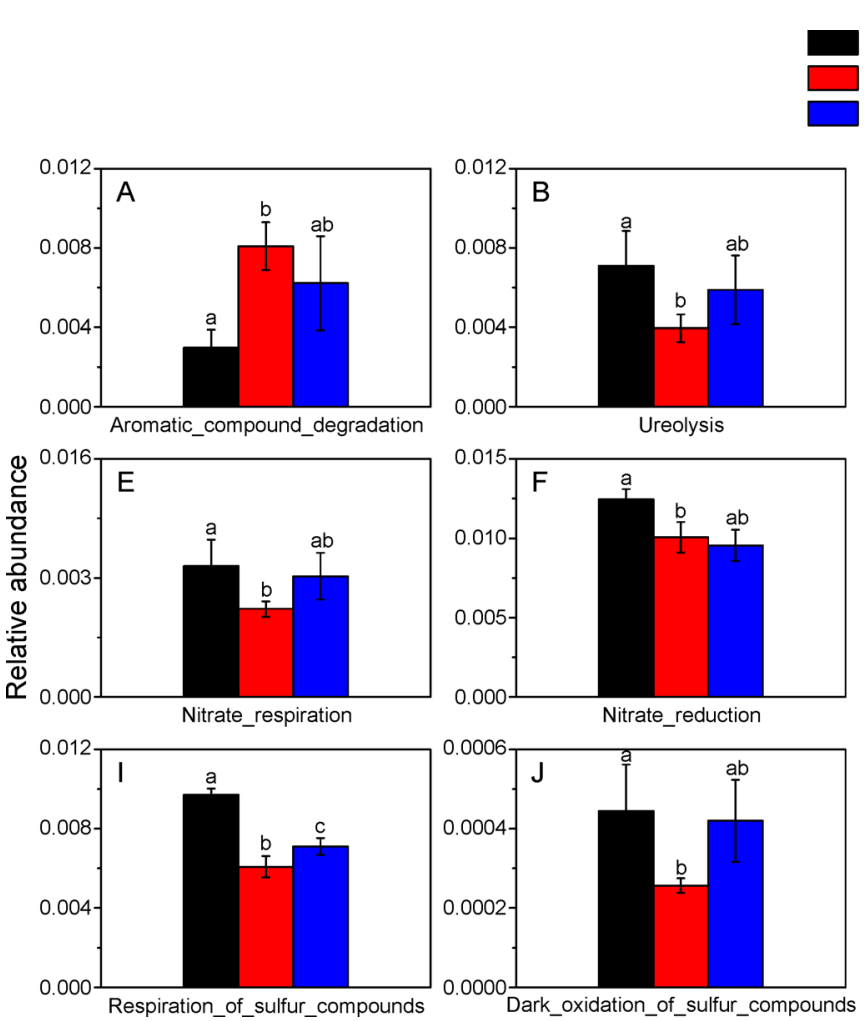

\section{Control \\ GO \\ RGO}

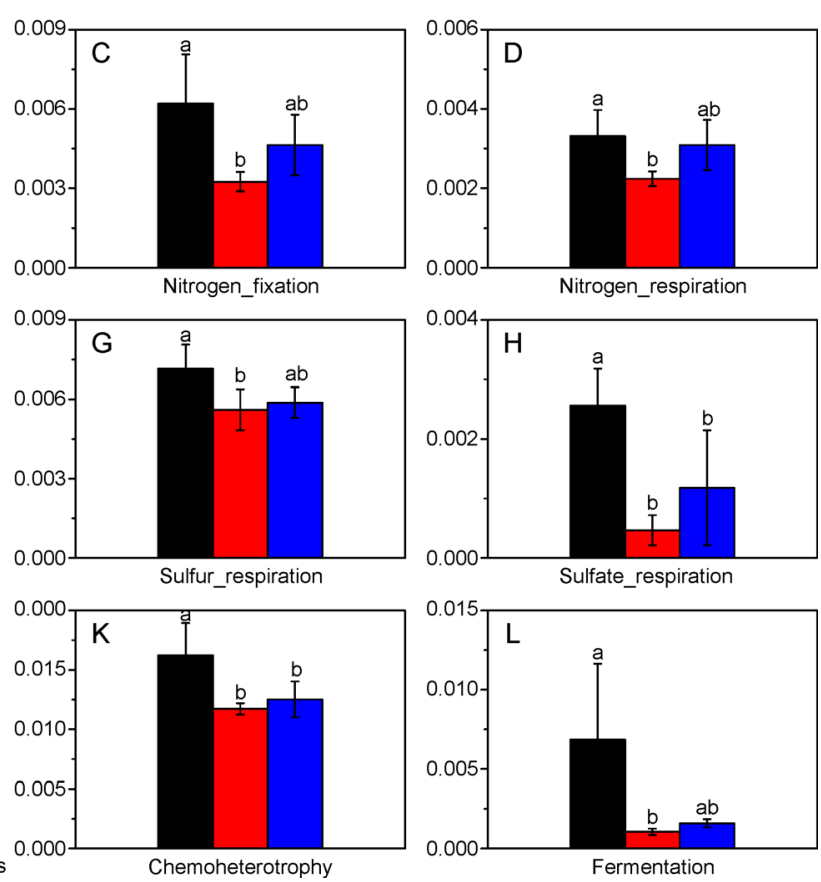

Fig. 2 Functional groups whose relative abundances exhibited significant differences between the control group and at least one treatment group ( $p<0.05$, using the least significant difference (LSD) test). The error bars on the results represent the means \pm standard deviation $(n=3)$. The different lowercase letters on the error bars indicate significant differences at $p<0.05$ (LSD test). 
and chemoheterotrophy. Once the exposure of GBNs changes microbial communities and functional groups, the cycling of biogeochemically reactive elements driven by these functional groups will be disturbed (Ge et al., 2018). Interestingly, "aromatic compound degradation" was the only functional group (Fig. 2 A) whose relative abundance increased due to either $\mathrm{GO}$ exposure $(p<0.05)$ or RGO exposure $(p>0.05)$. As GBNs contain a variety of aromatic hydrocarbon structures, they could selectively promote populations with the function of aromatic compound degradation (Mu et al., 2019). Our findings revealed that $\mathrm{GO}$ had stronger effects on bacterial community functions than RGO. However, the dominant bacterial communities were consistent at the genus level between GO and RGO (Fig. S4), which might suggest that a few key OTUs in the "GO" group dominated the functional groups regardless of OTU richness. Indeed, Chen et al. (2020) found that rare microbial taxa rather than the dominant taxa were the major drivers of multifunctionality in biological processes.

\section{Conclusions}

Our results demonstrated that RGO exerts stronger effects on alpha diversity and weaker effects on community structure and functional groups of soil bacterial communities than GO.

\section{Acknowledgments}

This work was financially supported by the National Natural Science Foundation of China (Grant No. 31600411) and High Quality Food Microbiology Curriculum of Shanxi Normal University (Grant No. 2017YZKC-24).

\section{Electronic supplementary material}

Supplementary material is available in the online version of this article at https://doi.org/10.1007/s42832-020-0043-5 and is accessible for authorized users.

\section{References}

Chen, Q.L., Ding, J., Zhu, D., Hu, H.W., Delgado-Baquerizo, M., Ma, Y. B., He, J.Z., Zhu, Y.G., 2020. Rare microbial taxa as the major drivers of ecosystem multifunctionality in long-term fertilized soils. Soil Biology \& Biochemistry 141, 107686.

Ge, Y., Shen, C.C., Wang, Y., Sun, Y.Q., Schimel, J.P., GardeaTorresdey, J.L., Holden, P.A., 2018. Carbonaceous nanomaterials have higher effects on soybean rhizosphere prokaryotic communities during the reproductive growth phase than during vegetative growth. Environmental Science \& Technology 52, 6636-6646.

Gibbons, S.M., 2017. Microbial community ecology function over phylogeny. Nature Ecology \& Evolution 1, 1-2.

Kah, M., Tufenkji, N., White, J.C., 2019. Nano-enabled strategies to enhance crop nutrition and protection. Nature Nanotechnology 14 , 532-540.

Louca, S., Parfrey, L.W., Doebeli, M., 2016. Decoupling function and taxonomy in the global ocean microbiome. Science 353,1272 1277.

Mu, L., Zhou, Q.X., Zhao, Y.J., Liu, X.W., Hu, X.G., 2019. Graphene oxide quantum dots stimulate indigenous bacteria to remove oil contamination. Journal of Hazardous Materials 366, 694-702.

Pulizzi, F., 2019. Nano in the future of crops. Nature Nanotechnology 14, 507-507.

Ren, X.Y., Zeng, G.M., Tang, L., Wang, J.J., Wan, J., Feng, H.P., Song, B., Huang, C., Tang, X., 2018. Effect of exogenous carbonaceous materials on the bioavailability of organic pollutants and their ecological risks. Soil Biology \& Biochemistry 116, 70-81.

Rodrigues, D.F., Jaisi, D.P., Elimelech, M., 2013. Toxicity of functionalized single-walled carbon nanotubes on soil microbial communities: implications for nutrient cycling in soil. Environmental Science \& Technology 47, 625-633.

Wang, J., Chen, Z.M., Chen, B.L., 2014. Adsorption of polycyclic aromatic hydrocarbons by graphene and graphene oxide nanosheets. Environmental Science \& Technology 48, 48174825.

Yin, J., Wang, Y., Gilbertson, L.M., 2018. Opportunities to advance sustainable design of nano-enabled agriculture identified through a literature review. Environmental Science: Nano 5, 11-26. 